\title{
EFFECT OF EXPERIMENTALLY INDUCED OTITIS MEDIA ON COCHLEAR IMPLANTS
}

\author{
BuRKhaRd K-H. FRANZ, MD, Dip AUD \\ Graeme M. Clark, PhD, fRacs \\ DAVID M. BLOOM, B APP SC
}

East melbourne, australia

\begin{abstract}
Cat cochleas implanted with scala tympani prostheses were investigated histologically after inoculating the bullae with a suspension of group A streptococci. The prosthesis was passed through the round window membrane in one ear. In the other the prosthesis bypassed the round window via an opening anteroinferior to the round window niche. Before death, horseradish peroxidase was administered as a tracer for possible pathways of infection. Results showed that group A streptococci were pathogenic to the cat and caused inflammation in the bulla. The unimplanted round window membrane and the seals around the electrode entry points prevented infection from entering the cochlea. The seals around electrodes inserted either through the round window membrane or an opening drilled anteroinferior to the niche were equally effective. The horseradish peroxidase tracer studies showed, however, that a gap existed between the electrode and membranous seal, and this could be a potentially vulnerable site under certain conditions. Drilling an anteroinferior opening into the cochlea resulted in bony sequestra entering the cochlea. This can be avoided by blue-lining the opening and removing bone with picks before making an opening through the endosteum.
\end{abstract}

KEY WORDS - cochlear implant, electrode insertion, group A streptococci, horseradish peroxidase, otitis media.

\section{INTRODUCTION}

It has been shown in animals and in humans that a prosthesis can be implanted into the cochlea without significant damage to the organ of Corti and auditory nerve fibers. ${ }^{1-7}$ In addition prophylactic antibiotic therapy and the laminar flow of sterile air are effective in preventing infection. ${ }^{8,9}$ Infection and surgical trauma nevertheless remain a potential hazard for nerve fiber survival.

A previous animal study that used horseradish peroxidase as a tracer demonstrated that the implanted round window membrane showed increased permeability for a period of approximately 2 weeks after surgery, ${ }^{10}$ and this indicates that middle ear infection during the period is a potential risk. Nevertheless a pilot study in our department prior to this investigation showed that the cat can be successfully inoculated with a suspension of group A streptococci and that the inner ear is not affected by the severe inflammation in the middle ear (W. Brennan, unpublished observations).

The objective of the present study was to provide further information on the morphologic changes in the implanted cochlea after inoculation with group A streptococci and to compare the seals produced by either inserting an electrode into the scala tympani through the round window membrane or through an opening anteroinferior to the round window niche, as these two surgical approaches are currently in use.

\section{METHODS AND MATERIALS}

Five otoscopically normal adult cats, weighing between 2.5 and $4 \mathrm{~kg}$ were used in this study. Anesthesia was induced with ketamine hydrochloride (Ketalar) $(10 \mathrm{mg} / \mathrm{kg}$, intraperitoneally) and Rompun $(0.5 \mathrm{mg} / \mathrm{kg}$, intraperitoneally), and surgery was performed under strict aseptic conditions.

In addition, one animal was used as a control where neither cochlea was implanted. In this animal the bullae were inoculated on both sides to determine the effects of a streptococcal infection on the intact round window membrane.

The right ears were implanted with a prosthesis that passed through a large incision close to the center of the round window membrane. The left ears were implanted through a hole drilled into the scala tympani of the basal turn at a point 2 to $3 \mathrm{~mm}$ anteroinferior to the round window. The opening was made using a $1.0-\mathrm{mm}$ diamond-paste bur. Suction irrigation was used, the bone was not blue-lined, and the endosteum was entered with the bur. The prostheses were inserted approximately 5 to $7 \mathrm{~mm}$ into the scala tympani.

Each Silastic prosthesis had a diameter of $0.6 \mathrm{~mm}$ and three platinum rings at its distal end, which were connected to Teflon-coated platinum wires. ${ }^{11}$ After implantation the cats received a daily dose of $125 \mathrm{mg}$ Amoxil (Beecham Research Labs, Clayton, Australia) over a period of 1 week.

The bullae were inoculated with $10^{7}$ microorganisms per milliliter in normal saline following recovery periods of 4 to 23 weeks after implantation. The quantity introduced into the bulla varied between 0.7 and $1.0 \mathrm{~mL}$. The opened bulla was filled with

From the Department of Otolaryngology, University of Melbourne, The Royal Victorian Eye and Ear Hospital, East Melbourne, Australia. This study was supported by the National Health and Medical Research Council of Australia and the Deafness Foundation of Victoria.

REPRINTS - B. K-H. Franz, MD, Dept of Otolaryngology, University of Melbourne, The Royal Victorian Eye and Ear Hospital, East Melbourne, Victoria 3002, Australia. 


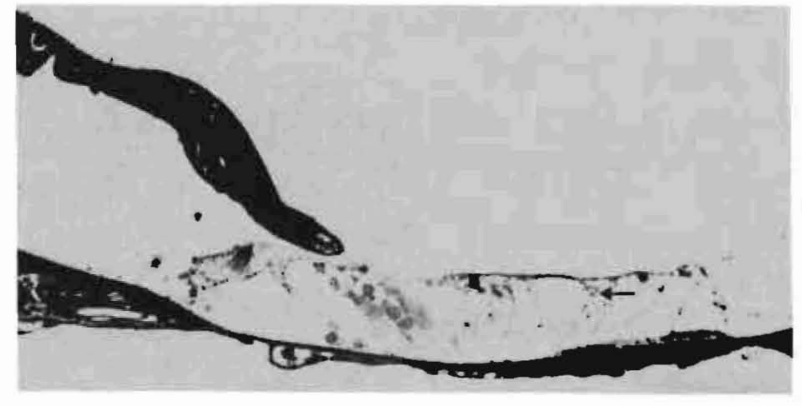

Fig 1. Photomicrograph of unimplanted cochlea following inoculation of group A streptococci and horseradish peroxidase into bulla showing particles of horseradish peroxidase (arrow) in organ of Corti.

Gelfoam before direct inoculation with a suspension of group A streptococci.

Before death each bulla was filled with $10 \% \mathrm{wt} / \mathrm{vol}$ horseradish peroxidase (Sigma Chemical Co, St Louis) for 15 minutes. Implanted cats were killed 3, 6, 7, and 9 weeks after inoculation. The nonimplanted cat was inoculated in the right ear 4 weeks before death and in the left ear 2 weeks before death.

The temporal bones were removed after killing, and the specimens fixed in $2 \%$ paraformaldehyde and $2.5 \%$ glutaraldehyde in $0.1 \mathrm{M}$ phosphate buffer for 3 to 5 hours, and then kept in fresh fixative overnight. The temporal bones were washed for 30 minutes in a saturated buffered solution ( $\mathrm{pH} \mathrm{7.6)} \mathrm{of}$ 3,3 diaminobenzidine (about $3 \mathrm{mg} / 10 \mathrm{~mL}$ ) with hydrogen peroxide added to make a final concentration of $0.01 \%$, then decalcified in a saturated solution of EDTA in 10\% neutral buffered formalin. After decalcification the specimens were washed in distilled water, dehydrated in ascending concentrations of acetone, and finally embedded in Spurr's resin.

Sections were cut at a thickness of $2 \mu \mathrm{m}$, and sections were collected every $130 \mu \mathrm{m}$ for staining. After mounting, Spurr's resin was removed from the sections with a saturated solution of sodium hydroxide in absolute alcohol. The sections were stained with hematoxylin and eosin, PAS, and methamine-silver; the latter was used to intensify the peroxidase reaction. Sections were examined using a light microscope.

\section{RESULTS}

The direct inoculation of suspensions of group A streptococci was successful in inducing inflammation in all cats. Histologic observations on the inoculated nonimplanted cat showed a chronic proliferative inflammation in the round window niche 2 weeks (left ear) and 4 weeks (right ear) after inoculation. The inflammation was characterized by proliferation of connective tissue cells and the presence of exudate. Although the bullae were inoculated 2 weeks apart, the inflammatory responses were histologically similar. The round window membranes had swollen to almost three times their normal thickness. The epithelia showed two or three layers. Ciliated cells appeared on the surface and were more concentrated at the periphery of the membrane. The subepithelial layer, however, contributed most to the increased thickness with a reactive connective tissue. The formation of new capillaries and accumulation of inflammatory cells with interstitial fluid were visible. In scattered areas small pockets of purulent exudate were found. The epithelial and subepithelial layers of the round window membrane were involved in forming protuberances that filled the round window niche. The endothelial layer appeared passive, and did not take part in this activity.

Horseradish peroxidase passed the epithelial layer of the membrane and was seen in the subepithelial layer within cells and in the intercellular spaces. Horseradish peroxidase appeared in the scala tympani and was observed in the organ of Corti (Fig l). Microorganisms were not seen. Swabs were taken from the inflamed area, but were negative for group A streptococci. The organ of Corti, nerve fibers, and ganglion cells appeared normal. The labyrinth did not respond to the severe inflammation outside the cochlea.

In the ears of the four cats implanted with a pros-

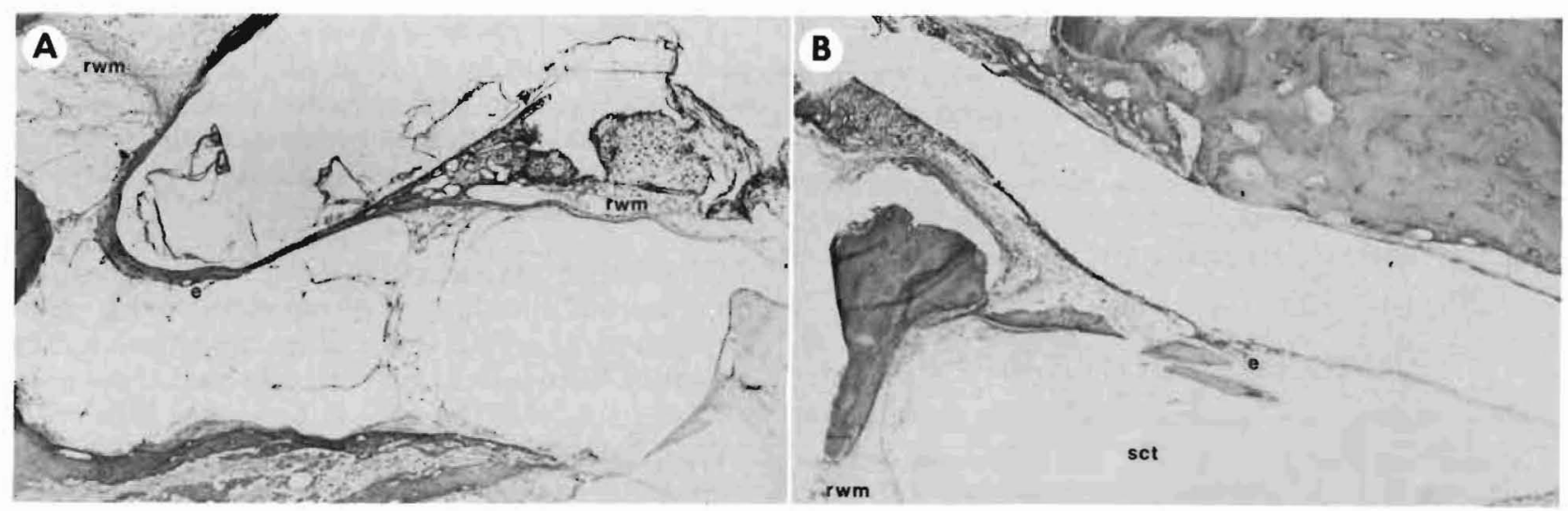

Fig 2. Photomicrograph showing implanted cochlea with A) prosthesis passed through round window membrane and B) prosthesis that bypassed round window membrane. sct - scala tympani, rwm - round window membrane, e - envelope. 
thesis that passed through the round window membrane into the scala tympani (Fig 2A), the inflammation in the round window varied from subacute to chronic. The transition stage between acute and chronic inflammation in which exudation and proliferation were counterbalanced, was classified as a subacute inflammation.

The inflammatory response was always more severe close to the prosthesis, and here the round window membrane was slightly thicker than elsewhere. The main areas of activity were seen in the epithelial and subepithelial layers. The epithelium was two or three cells thick, and the subepithelium showed an increase in interstitial fluid and connective tissue. The envelope that surrounded the prosthesis was also involved in the inflammatory response.

The organ of Corti appeared complete, and nerve fibers were preserved. In one case, however, where the prosthesis fractured the spiral lamina, there was localized collapse of the organ of Corti, restricted nerve fiber loss, and new bone formation.

Horseradish peroxidase passed into the subepithelial intercellular spaces of the round window membrane, but its concentration diminished rapidly toward the deeper portions of the membrane. Similar observations were made in the envelope. Horseradish peroxidase passed the gap between the prosthesis and the round window membrane into the envelope, and from there into the scala tympani. The organ of Corti showed traces of horseradish peroxidase similar to the unimplanted cat.

In the left ears of the four cats that were both implanted with a scala tympani prosthesis that bypassed the round window (Fig 2B) and successfully inoculated with group A streptococci, the inflammatory reactions of the mucosa close to the prostheses were classified as chronic. The round window membrane was also inflamed, but not as severely as in the immediate neighborhood of the prosthesis. The envelopes of the prostheses were again thick, and showed an organized layer of connective tissue around them.

Surgical trauma was prominent in these cochleas. New bone growth was associated with fractures in the spiral lamina and modiolus, and bone chips were seen in the scala tympani scattered within the basal turn. The organ of Corti showed hair cell losses and collapsed supporting cells. Nerve fiber losses were observed to be always associated with fractures and were strictly localized.

Horseradish peroxidase entered the basal turn of the cochlea. It passed through the gap between the prosthesis and the bony wall of the cochlea, and from there via the envelope into the scala tympani, Hardly any traces were found in the organ of Corti. Horseradish peroxidase entered the cells in the round window membrane by passing through the epithelial layer, but particles were not seen in intercellular spaces.

\section{DISCUSSION}

In our pilot study prior to this investigation it was found that a suspension of group A streptococci could successfully be used for inoculation studies in the cat (W. Brennan, unpublished observations). Furthermore, a severe chronic inflammation in the bulla and middle ear was not associated with damage to important structures of the cochlea. The implanted cochlea also demonstrated an effective defense system similar to the unimplanted one. The implanted cochlea per se was not at a disadvantage.

Although promising, these findings do not allow the conclusion that infection is without danger to the implanted cochlea. For example, an increased permeability of the round window membrane has been observed in otitis media and could lead to an associated sensorineural hearing loss. ${ }^{12}$ Furthermore, the round window membrane was found to be an important avenue of entry for toxins into the inner ear. ${ }^{13}$

However, despite the assumption that infection of the middle ear causes increased permeability of the round window membrane, and thus vulnerability of the inner ear structures, there are indications that this hypothesis is an oversimplification. In a clinical review a correlation between middle ear disease and cochlear dysfunction was not evident. ${ }^{14}$

The present study, although showing the ease with which horseradish peroxidase entered the scala tympani when inflammation was present in the bulla, does not establish that this increased permeability of the round window membrane or electrode envelope leads to the spread of infection to the inner ear or that there is any difference between the unimplanted and implanted round window membranes. This conclusion is supported by the absence of inflammatory changes in the cochleas of the inoculated bones. The results, however, must still be interpreted with some reservation. For example, the animals were inoculated 4 to 23 weeks after implantation, so that the electrode entry points should have healed, and only one pathogenic organism was used in the study.

Apart from these reservations about biologic safety, this study also showed that trauma can be a hazard in cochlear implant surgery as demonstrated by other investigators. ${ }^{1,3.4 .7 .15}$ However, the trauma seen in the cat cochlea when an electrode was inserted through an anteroinferior opening was due to the fact that the scala tympani has a relatively larger hook region. It was therefore more difficult to insert an electrode along the basal turn through an anteroinferior opening than via the round window. The reverse applies in humans, where an anteroinferior opening avoids the hook region and makes it easier to insert the electrode without trauma. Alternative- 
ly, if the electrode is inserted through the round window, it is important to remove the anteroinferior overhang and drill away the crista fenestrae. ${ }^{6}$

This experimental study on cats showed that bone chips, produced by drilling, can be introduced into the cochlea and included in the fibrous tissue surrounding the electrode. Although they did not result in any tissue reaction, infected bone chips can become a problem in healing and are a concern in surgery. ${ }^{16}$ For this reason it is good practice when making an anteroinferior opening into the cochlea to blue-line the bone and then remove the necessary bone with picks before penetrating the endosteal lining. Similarly, care must be taken when drilling the crista fenestrae in a round window approach to use suction irrigation.

\section{CONCLUSION}

The cat cochlea implanted with a scala tympani prosthesis via the round window membrane or an opening anteroinferior to the round window niche demonstrated resistance to infection similar to the nonimplanted cochlea. The inflammatory response to infection also involved the envelope that formed as an organized layer of connective tissue around the prosthesis. Horseradish peroxidase passed the gap between the prosthesis and the round window membrane into the envelope and from there into the scala tympani. An absolute seal was not obtained.

ACKNOWLEDGMENTS - We thank Dr R. Robins-Browne from the Microbiology Department of the University of Melbourne as well as R. Shepherd and $P$. Busby for helpful discussions.

\section{REFERENCES}

1. Clark GM, Kranz HG, Minas H, Nathar JM. Histopathological findings in cochlear implants in cats. J Laryngol Otol 1975;89:495-504.

2. House WF, Berliner KI, Eisenberg LS, Edgerton BJ, Thielmeir MA. The cochlear implant: 1980 update. Acta Otolaryngol (Stockh) 1981;91:457-62.

3. Merzenich MM, Rebscher SJ, Loeb GE, Byers CL, Schindler RA. The UCSF cochlear implant project. Adv Audiol 1984;2: 119-44.

4. Shepherd RK, Clark GM, Pyman BC, Webb RL. Banded intracochlear electrode array: evaluation of insertion trauma in human temporal bones. Ann Otol Rhinol Laryngol 1985;94:55-9.

5. Clark GM, Pyman BC, Webb RL, Franz BK-HG, Redhead TJ, Shepherd RK. Surgery for the safe insertion and reinsertion of the banded electrode array. In: Clark GM, Busby PA, eds. International Cochlear Implant Symposium and Workshop Melbourne, 1985. Ann Otol Rhinol Laryngol 1987;96(suppl 128):10-2.

6. Franz BK-HG, Clark GM. Refined surgical technique for insertion of banded electrode array. In: Clark GM, Busby PA, eds. International Cochlear Implant Symposium and Workshop - Melbourne, 1985. Ann Otol Rhinol Laryngol 1987;96(suppl 128):15-7

7. Schindler RA. The cochlear histopathology of chronic intracochlear implantation. J Laryngol Otol 1976;90:445-57.

8. Clark GM, Pyman BC, Pavillard RE. A protocol for the prevention of infection in cochlear implant surgery. J Laryngol Otol 1980;94:1377-86.
9. Clark GM, Dowell RC, Pyman BC, et al. Clinical trial of multichannel cochlear prosthesis: results on ten postlingually deaf patients. Aust NZ J Surg 1984;54:519-26.

10. Franz B, Clark GM, Bloom DM. Permeability of the implanted round window membrane in the cat. An investigation using horseradish peroxidase. Acta Otolaryngol [Suppl] (Stockh) 1984(suppl 410):17-23.

11. Patrick JF, Crosby PA, Hirshorn MS, et al. The Australian multi-channel implantable hearing prosthesis. 10th Anniversary Conference on Cochlear Implants: An International Symposium, UCSF 1983. In: Schindler, RA, Merzenich MM, eds. Cochlear implants. New York: Raven Press, 1985:93-100.

12. Goycoolea MV, Paparella MM, Goldberg B, Carpenter A-M. Permeability of the round window membrane in otitis media. Arch Otolaryngol 1980;106:430-3.

13. Schachern PA, Paparella MM, Goycoolea M, Goldberg B, Schlievert $\mathrm{P}$. The round window membrane following application of staphylococcal exotoxin: an electron microscopic study. Laryngoscope 1981;91:2007-16.

14. Dumich PS, Harner SG. Cochlear function in chronic otitis media. Laryngoscope 1983;93:583-6.

15. Sutton D, Miller JM, Pfingst BE. Comparison of cochlear histopathology following two implant designs for use in scala tympani. Ann Otol Rhinol Laryngol 1980;89(suppl 66):11-4.

16. Burri C. Posttraumatische Osteitis. Bern: Verlag Hans Huber, 1974. 


\section{University Library}

\section{- M M I E E R VA A gateway to Melbourne's research publications}

Minerva Access is the Institutional Repository of The University of Melbourne

Author/s:

Franz, Burkhard K-H.;Clark, Graeme M.;Bloom, David M.

Title:

Effect of experimentally induced otitis media on cochlear implants

Date:

1987

Citation:

Franz, B. K., Clark, G. M., \& Bloom, D. M. (1987). Effect of experimentally induced otitis media on cochlear implants. Annals of Otology, Rhinology \& Laryngology, March-April, 96(2), 174-177.

Persistent Link:

http://hdl.handle.net/11343/27249 